\title{
PEGylation Greatly Enhances Laccase Polymerase Activity
}

\author{
Jing $\mathrm{Su}_{1}{ }^{[\mathrm{a}, \mathrm{b}]}$ Jennifer Noro, ${ }^{[\mathrm{b}]}$ Ana Loureiro, ${ }^{[\mathrm{b}]}$ Madalena Martins, ${ }^{[\mathrm{b}]}$ Nuno G. Azoia, ${ }^{[\mathrm{b}]}$ Jiajia Fu, ${ }^{[\mathrm{a}]}$ \\ Qiang Wang, ${ }^{[a]}$ Carla Silva, ${ }^{[b]}$ and Artur Cavaco-Paulo*[a,b]
}

\begin{abstract}
Laccase catalyzes the oxidation and polymerization of phenolic compounds in the presence of oxygen. Herein, we report for the first time that a previous PEGylation of laccase enhances the polymerase activity 3 -fold compared with the reaction of the native enzyme, as confirmed by UV/Vis spectroscopy. The polymerization of catechol increased only 1.5 -fold if poly(ethylene glycol) (PEG) was added to the medium reaction.
\end{abstract}

Molecular-dynamics simulations suggest the formation of a miscible complex of polycatechol and PEG, which is responsible to push the reaction forward. In a negative control experiment set, all catalysts were entrapped inside polyacrylamide gels and here the native laccase showed a relatively higher activity. These results suggest that the mobility of PEG is a key feature for the enhancement of the reaction.

\section{Introduction}

Laccases have been investigated for their ability to catalyze the oxidation of various substrates such as phenols, aminol compounds, and their derivatives. These enzymes are mostly considered as extracellular proteins that are fairly stable if kept at neutral $\mathrm{pH}$ and under room temperature, with a broad substrate specificity, catalytic activity, effective $\mathrm{pH}$ range, and working temperature range. They have received much attention for their potential use in a wide variety of applications such as biosensors, bioremediation, green synthesis, green biodegradation of xenobiotics, and other areas. ${ }^{[1]}$

Recently, phenolic polymers have attracted an increasing attention as novel materials based on their excellent properties and applications in several areas. The enzymatic synthesis using laccase under mild reaction conditions has been considered as an alternative process for the polymerization of phenolic polymers replacing the use of toxic chemicals. ${ }^{[2-5]}$

The control of the chemical structure of polymers obtained by enzyme-catalyzed template polymerization has also been studied. Generally, template polymerization is defined as a process in which the monomer units are organized by interactions with a template macromolecule. ${ }^{[6]}$ Walde and Guo considered the template as a type of additive that helps controlling the

[a] J. Su, Dr. J. Fu, Prof. Q. Wang, Prof. A. Cavaco-Paulo International Joint Research Laboratory for Textile and Fiber Bioprocesses Jiangnan University Wuxi 214122 (China)

E-mail:artur@deb.uminho.pt

[b] J. Su, J. Noro, Dr. A. Loureiro, Dr. M. Martins, Dr. N. G. Azoia, Dr. C. Silva, Prof. A. Cavaco-Paulo

Centre of Biological Engineering

University of Minho Campus de Gualtar, 4710-057, Braga (Portugal)

(D) The ORCID identification number(s) for the author(s) of this article can be found under https://doi.org/10.1002/cctc.201700849.

2. This publication is part of a Virtual Issue on the Valorization of Lignocellulosic Biomass, which comes as part of a European COST Action. More information regarding this Virtual Issue can be found on the ChemCatChem homepage. outcome of a polymerization reaction..$^{[7]}$ The chemical structure of the polymers obtained through enzyme-catalyzed reactions can be controlled by the addition of chemical structure-controlling templates. Two general strategies concerning the mode of action of templates during polymerization were proposed: a) the direct interaction between the template and the reacting monomers and/or the growing polymer chains and b) the structuring of the reaction medium in such a way that the polymerization reaction takes place spatially confined. In the first case, the reaction is called "template-assisted" polymerization. The monomers and the growing polymer chains are assumed to interact with the template, which can influence the chemical structure of the polymers obtained, for example, increasing reaction regioselectivity. In the second case, the reaction system is divided in two regions by the template: in one region, the monomers are soluble and undergo polymerization, and in the other region, the monomers and the growing polymer chains are not soluble and less accessible to the enzyme. The templates affect the morphology of the polymers obtained, namely, the particle geometry and size, porosity, and chemical structure. Kim and co-workers suggested that the presence of the template can influence the polymerization rate of the monomer compared with the blank polymerization and also the properties of the final products, such as the molecular weight and the stereoregularity. ${ }^{[2,8]}$ The enzymatic polymerization of phenolic substrates by horseradish peroxidase was studied by using poly(ethylene glycol) (PEG) as template in an aqueous medium. The addition of the additive produced a miscible complex of polyphenol and PEG as precipitates in high yields. The formation of a phenol-PEG complex was verified by hydrogen-bonding interaction. It was confirmed that the amount of PEG strongly affected the polyphenol yield. ${ }^{[2]}$ These authors also studied the enzymatic oxidative polymerization of phenol by the addition of poly(ethylene glycol) monododecyl ether (PEGMDE) template to the medium. They observed that the addition of this additive pushed forward the polymerization in water. ${ }^{[1,9]}$ The presence of PEGMDE template 
in the aqueous medium greatly improved the regioselectivity, yielding polyphenol with a phenylene unit content near $90 \%$. Steevensz et al. showed that PEG extended the lifetime conversion of the phenolics into polymers. ${ }^{[1]}$ If a "PEG effect" was observed, there was a linear relationship between the amount of additive used and the amount of precipitate, suggesting a strong interaction between products and PEG, confirmed by NMR spectroscopy. PEG only enhances the laccase-catalyzed oxidative coupling of certain substrates, depending on the substituent positioning of the substrate and the functionality of the polymers formed. ${ }^{[10]}$

Modaressi and co-workers examined the feasibility of using laccase to treat synthetic wastewater containing bisphenol-A (BPA) as PEG was thought to reduce enzyme inactivation. ${ }^{[11]}$ Its addition to the medium had a significant protective effect on the activity of laccase. It is inferred that an interaction between PEG and the polymeric products resulted in the protection of the enzyme (Scheme 1). However, the mechanism of enzyme protection by PEG need to be studied in depth.

(a)



(b)

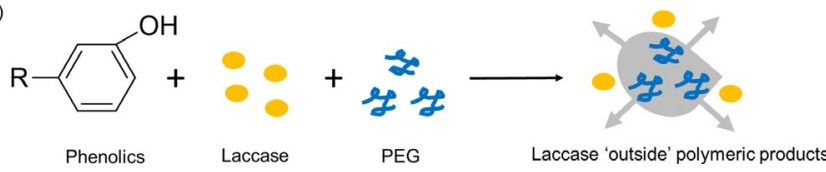

Scheme 1. Proposed mechanism for laccase polymerization (a) in the absence and (b) in the presence of PEG. ${ }^{[12]}$.

The polymerization process was investigated by using PEG with different molecular weight. ${ }^{[13,14]}$ Studies with laccase demonstrated that the effectiveness of PEG as a protective additive was dependent on its molecular weight. By using low-molecular-weight PEG, an effective complex with polymeric products could not be accomplished because of the biodegradation of PEG. In contrast, high-molecular-weight PEG gave rise to an increase of the solution viscosity hindering polymer production. ${ }^{[2]}$ PEG with a molecular weight between 3000 and 4000 Da was revealed as the most effective and environmentally friendly alternative, ${ }^{[15]}$ protecting laccases from entrapment inside the formed polymers. It has been hypothesized that PEG attaches to phenolic polymers forming a miscible complex and the enzyme remains in the solution to proceed polymerization, as proposed in Scheme 1.

In the present work, we study the role of PEG as a template on the laccase-assisted polymerization of catechol. To achieve this, the enzymatic polymerization of the phenolic monomer was conducted by using different conditions, namely, a) in the absence of PEG; $b$ ) in the presence of PEG, and c) with a previously PEGylated laccase. The produced polymers were characterized by matrix-assisted laser desorption/ionization time-offlight mass spectrometry (MALDI-TOF). UV absorbance spectra were taken for the analysis of the color change during polymerization. The immobilization of all catalysts on acrylamide gels was performed to test the reaction under reduced mobility of PEG. Molecular dynamic (MD) simulations were conducted to understand the role of PEG during laccase-assisted polymerization of catechol.

\section{Results and Discussion}

\section{PEGylation of laccase}

PEGylation is the covalent attachment of one or more molecules of PEG to a protein. PEGylation can improve thermal stability and reduce deactivation of the enzyme, and increase the biocatalyst lifetime. In this work, the PEGylation of laccase was performed and confirmed by sodium dodecyl sulfate polyacrylamide gel electrophoresis (SDS-PAGE, Figure 1). The gel was stained with silver for protein detection (Figure 1). The silver staining shows a smear between 100-180 kDa for PEGylated samples (area in the red rectangle), confirming the increase of the molecular weight corresponding to the presence of the PEG.

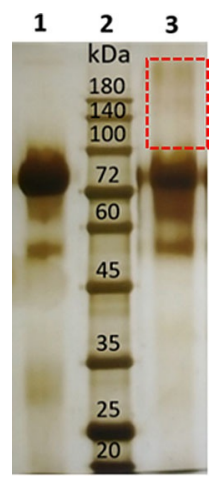

Figure 1. SDS-PAGE analysis of laccase after PEGylation with 20 kDa PEG, using silver staining for protein detection; native laccase $5 \mu$ (lane 1), GRS protein Marker Blue standards $2.5 \mu \mathrm{g}$ (lane 2), PEGylated laccase $5 \mu \mathrm{g}$ (lane 3).

\section{Laccase-assisted polymerization of catechol in the presence of PEG}

From previous studies, it is known that in normal cases native laccase produced a low amount of polymer and gave rise to low polymerization degrees. This is attributed to the interactions between laccase and the reaction products leading to enzyme inactivation. PEG is considered as a particularly effective additive for laccase protecting it against inactivation. Some reports have described the successful use of PEG to enhance the formation of polymers during the polymerization process. ${ }^{[7]}$ Herein, a series of experiments were conducted to analyze the role of PEG during catechol polymerization. The laccase-assisted polymerization of catechol was performed in acetate buffer ( $\mathrm{pH} \mathrm{5):} \mathrm{a)} \mathrm{in} \mathrm{the} \mathrm{absence} \mathrm{of} \mathrm{PEG,} \mathrm{b)} \mathrm{in} \mathrm{the} \mathrm{pres-}$ ence of PEG (3-4 kDa), and c) with PEGylated laccase (20 kDa), 
by using air oxygen as oxidizing agent. During the reaction, oligomers and polymers are formed until their solubility limit is reached, and the powdery precipitates were collected by centrifugation.

The UV absorbance of polycatechol in absence and in the presence of PEG in acetate buffer $(\mathrm{pH} 5)$ is shown in Figure 2. By the addition of PEG to the system, higher values of

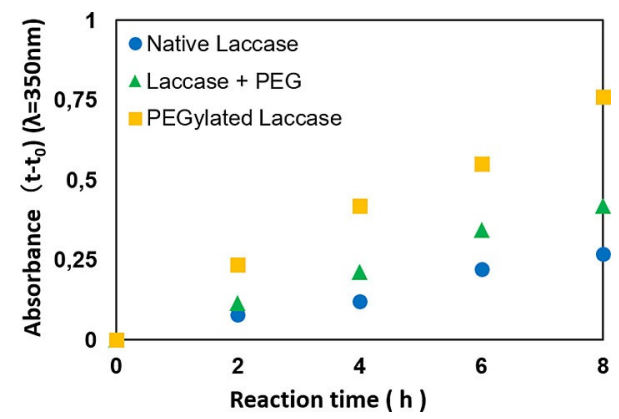

Figure 2. UV absorption at $350 \mathrm{~nm}$ during the polymerization of catechol under different conditions.

absorbance at $350 \mathrm{~nm}$ are detected (2-fold increase), especially if PEGylated laccase is used (3-fold of color increase). The differences of intensity are related with different polymerization degrees resulting from the differentiated hydrogen-bonding interactions between the hydroxyl groups of catechol and PEG, which are suggested to occur through a "zip mechanism". ${ }^{[2,7]}$ After laccase addition, the reaction mixtures rapidly changed from colorless to brown or dark brown owing to the quinones formed during oxidation. The color differences can be attributed to the amount of soluble oligomers in the reaction superna$\operatorname{tant}^{[16]}$ resulting from the presence of PEG in the medium. A high polymerization degree is obtained if PEG is present, even in the free form of linked to laccase (average degree of polymerization, DP, of 8, Table 1). This is a step further from the reported results that higher DPs were obtained only after the addition of an additive to the medium. ${ }^{[2]}$ PEGylation of laccase is responsible not only for a higher degree of polymerization, but also for the production of a higher amount of polycatechol evidenced by the amount of powder obtained after freeze-drying (data not shown).

It has been established that the PEG effect is less pronounced if crude enzymes are used. ${ }^{[1]}$ Herein, we used a commercial native laccase obtained from Novozymes, which contained specific additives and stabilizers that could also contribute for the differences observed between the samples containing different amounts of PEG. After PEGylation, the PEGylated protein was ultrafiltrated to eliminate a large amount of the stabilizers and obtain a more pronounced PEG effect. Our results also suggest that the interaction of the additive with the enzyme may contribute for the PEG effect. A robust and less inactivated enzyme is obtained after PEGylation, which explains the high amount of polymer after the reaction.

\section{Enzymatic polymerization of catechol in the presence of im- mobilized laccase onto acrylamide gels}

The use of laccases in practical industrial applications is still limited because of their high cost and low stability. ${ }^{[17]}$ To overcome these limitations, the immobilization of laccase has been studied, and many immobilization methods have been reviewed. ${ }^{[14]}$ Several techniques are applied during the immobilization procedure based on chemical and physical mechanisms such as cross-linking, adsorption, entrapment, and encapsulation. One of the most widely used systems of laccase immobilization is the entrapment in a polymer lattice, polyacrylamide gel, obtained by polymerization/cross-linking of acrylamide. This type of immobilization has proved to be a particularly easy and effective way to immobilize enzymes or other proteins. $^{[18]}$

Herein, polyacrylamide gels were prepared to serve as supports for laccase and PEGylated laccase immobilization. Three different experiments were performed as described above. Native laccase, native laccase + PEG, and PEGylated laccase were immobilized during polyacrylamide gels production and used for the polymerization of catechol (Figure 3). UV absorbance data acquired after polymerization revealed that PEG did not display the same template role as in the experiments in solution. If the catalyst was immobilized into acrylamide gels, the addition of PEG did not favor enzyme mobility hindering reaction progression and resulting in lower polymerization degrees (Figure 3).

The restrictions imposed by this three-dimensional network structure might constrain enzyme mobility and block reaction progression. If entrapped inside the gel, PEG cannot work as a template and forms the typical complexes with the polymer (Figure 4). These results indicate that mobility is an important feature in systems using this additive as a template for phenolics polymerization.

The mechanism of catechol polymerization by laccase has been proposed by others ${ }^{[19]}$ and defines repeating units of oxyphenylene after the reaction. To confirm the PEG role as a template, free in solution or chemically bond to laccase, we

\begin{tabular}{|c|c|c|c|c|c|c|}
\hline & $\begin{array}{l}\text { Protein conc. } \\
{\left[\mathrm{mg} \mathrm{mL}^{-1}\right]}\end{array}$ & $\begin{array}{l}\text { Specific activity } \\
{\left[\mathrm{Umg}_{\text {protein }}{ }^{-1}\right]}\end{array}$ & $\begin{array}{l}\text { OD after } 8 \mathrm{~h} \\
{[350 \mathrm{~nm}]}\end{array}$ & $\begin{array}{l}\text { Free } \mathrm{OH} \\
\text { [catechol as 1.00] }\end{array}$ & $M_{\mathrm{n}^{\prime}} M_{\mathrm{w}} P d^{[\mathrm{a}]}$ & Average DP \\
\hline Native laccase & & 6.50 & 0.268 & 0.498 & $748,776,1.03$ & 7 \\
\hline Laccase + PEG & 98 & 6.50 & 0.420 & 0.414 & $831,850,1.02$ & 8 \\
\hline PEGylated laccase & & 2.11 & 0.760 & 0.329 & $833,849,1.02$ & 8 \\
\hline
\end{tabular}




\section{PEG-controlled polymerization of catechol}

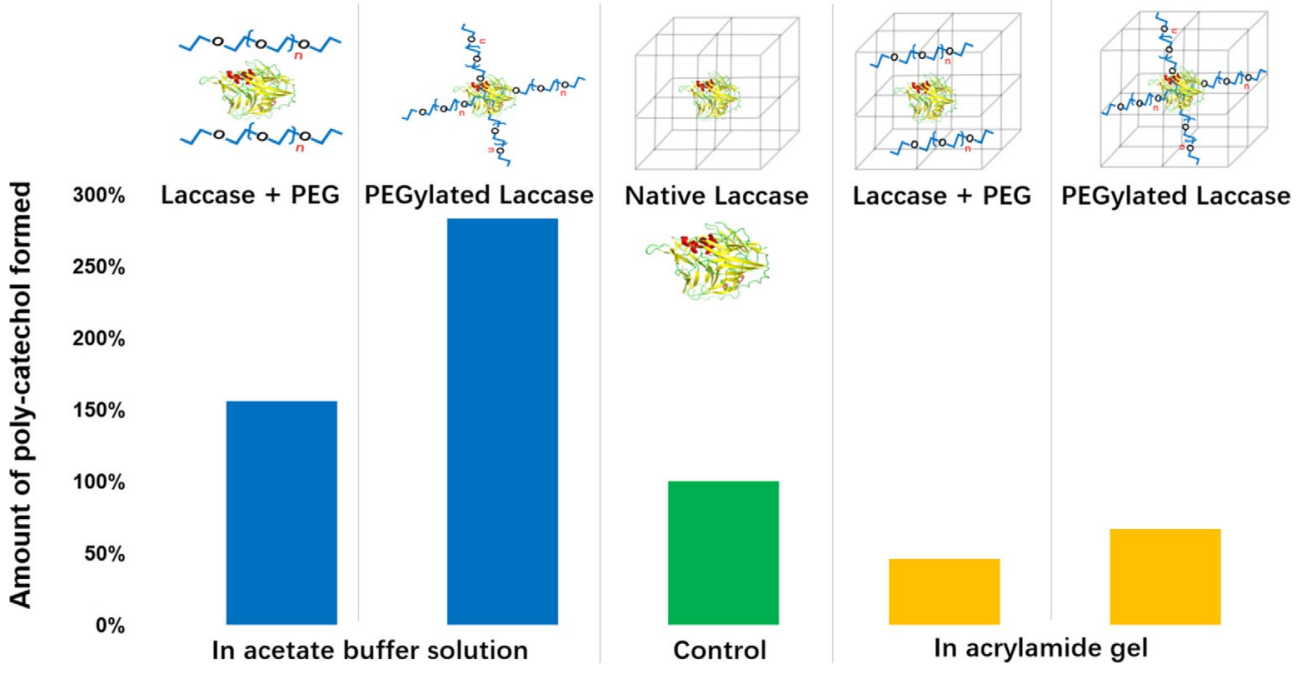

Figure 3. UV absorbance (\%) of polycatechol polymerized by laccase in solution (blue) and by laccase immobilized onto polyacrylamide gels (yellow) for $8 \mathrm{~h}$ $(\lambda=350 \mathrm{~nm}$; control: native laccase in solution and in gel corresponds to $100 \%$ absorbance, green).

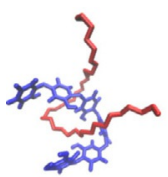

(a)

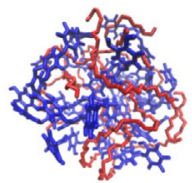

(b)

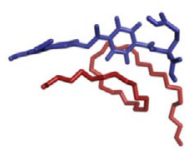

(c)



(d)
Figure 4. MD simulations of catechol and MTX polymerization showing (a) 6 repeating units of polycatechol (in blue) and 12 repeating units of PEG (in red); (b) final miscible complex between polycatechol and PEG; (c) 1 unit of MTX (in blue) and 12 repeating units of PEG (in red); (d) final non miscible complex between polyMTX and PEG.

estimated the amount of oxyphenylene units by measuring the total content of free $\mathrm{OH}$ in all the mixtures after the reaction (Table 1). The Folin-Ciocalteau method measures the total content of $\mathrm{OH}$ after catechol polymerization, and the values are normalized by considering the total content of free $\mathrm{OH}$ of catechol as 1 (100\%). From Table 1, a decrease of the total content of free $\mathrm{OH}$ after catechol polymerization in the presence of PEG can be observed, which was more pronounced for samples polymerized by PEGylated laccase. Because the content of $\mathrm{OH}$ groups decreases as more chains of oxyphenylene are present, this method confirms higher polymerization degrees. From Table 1, one can highlight the high stability of PEGylated laccase during polymerization. A lower specific activity gave rise to higher polymerization yields than in the case if non-PEGylated laccase was applied. PEGylation played here a crucial role on protecting the enzyme for deactivation allowing PEG to work as a reaction template. The data also revealed that laccase PEGylation would be a strategic tool to improve reaction yields as confirmed by the absorbance data and the total content of free $\mathrm{OH}$ (Table 1). Moreover, the processing drawbacks related with the enzyme cost can be overcome by a 3-fold reduction of enzyme needed to proceed the reaction and reach similar or higher conversion levels.
Molecular modelling simulations were performed to predict the interaction between PEG/polycatechol and PEG/methotrexate (MTX) at a molecular level. Polycatechol model with six repeating units (in blue) and a PEG model (in red) with 12 units were used for simulations (Figure $4 \mathrm{a}$ ). The methotrexate model with 1 unit (in blue) and 12 units of PEG (in red) were also used for simulations (Figure 4c). The images obtained resulted from a short MD simulation performed with GROMACS, using gromos54a7 force filed in water. Polycatechol demonstrates the ability to interact with PEG, forming miscible complexes, as depicted in Figure $4 \mathrm{~b}$ ). These results corroborate the experimental data, by which a 2-fold improvement of DP was observed if PEG was present on the system, preserving the enzyme from inactivation. The enzyme is therefore able to proceed reaction in solution. If the PEGylated enzyme is used, one can expect that the complex formed between PEG and the products of polymerization would remain close to the catalyst providing a protective effect without compromising its catalytic behavior. Moreover, the products of polymerization in the presence of PEG are inert to the enzyme and less accessible in solution, restraining its polymerization for higher DPs. This is evidenced by the formation of a higher amount of products after polymerization in the presence of PEG. The use of this additive as a template does not correspond necessarily to higher DPs but is the key factor to obtain higher yields of polymerization and an increased amount of polycatechol.

To predict if PEG would serve as template for other enzymatic-assisted polymerization reactions, we investigated the potential of $\alpha$-chymotrypsin (from bovine pancreas) for the polymerization of MTX in the presence of this additive. This serine protease has been described to catalyze the oligomerization of dipeptides in aqueous media. ${ }^{[20]}$ The results achieved if MTX was polymerized using native $\alpha$-chymotrypsin revealed a DP $=$ 5 with polymerization yield: $\mathrm{y}=80 \%$. The role of PEG as a template of MTX polymerization was also evaluated. To achieve 
Table 2. The role of PEG in the polymerization of MTX and catechol with different substrates using $\alpha$-chymotrypsin and laccase, respectively. ${ }^{[a]}$

\begin{tabular}{|llllll} 
& & $\begin{array}{l}\text { Enzyme } \\
{[\%(w / v)]}\end{array}$ & $\begin{array}{l}\text { PEG } \\
{[\mathrm{kDa}]}\end{array}$ & $\begin{array}{l}\text { DP } \\
\text { Relative conversion } \\
\text { yield [\%] }\end{array}$ \\
\hline 1a) & $\alpha$-chymotrypsin +MTX & 10 & - & 5 & 100 \\
1b) & PEGylated $\alpha$-chymotrypsin + MTX & 10 & 5 & 4 & 57 \\
2a) & Laccase + catechol & 20 & - & 7 & 100 \\
2b) & Laccase + PEG + catechol & 20 & $3-4$ & 8 & 150 \\
2c) & PEGylated laccase + catechol & 20 & 5 & 8 & 270 \\
\hline
\end{tabular}

[a] DP was calculated by MALDI-TOF analysis; relative polymerization yield was calculated as OD increase relatively to control for samples polymerized by laccase; for samples polymerized by chymotrypsin the yield was obtained by the quotient between the number of moles of the isolated polymer and the number of moles of the monomer)

this, $\alpha$-chymotrypsin was PEGylated with mPEG $(5 \mathrm{kDa})$ and further used for MTX polymerization. ${ }^{[21]}$ From the results obtained, no differences in DP and amount of polymer were observed after polymerization with PEGylated $\alpha$-chymotrypsin (Table 2; $1 \mathrm{a})$ and $1 \mathrm{~b})$ ). The molecular-modelling simulations also corroborate these findings, showing that polyMTX does not complex with PEG (Figure 4d).

These results indicate that different enzymes present variations in their affinities for the polymeric precipitate as well as in the sensitivity to additives such as PEG. Moreover, the different hydrophobicities of the polymer precipitates are modulated by the nature of the initial monomers. A product-additive relationship may be related with the functionality and position of the substituents of the substrate used. ${ }^{[1]}$ Also, the optimal PEG $M_{w}$ would vary between enzymes, owing to inactivation phenomena or to the nature of the products that interact with the enzymes during reaction, which are different among experiments and may alter the role of PEG as a template of the polymerization reaction. ${ }^{[1]}$

\section{Characterization of the polymers}

The enzymatic-assisted polymerization was confirmed by MALDI-TOF analysis (Figure 5). The collected data reveal peaks of polycatechol corresponding to average polymerization

degrees of 7, 8, and 8, if laccase, "laccase + PEG", and "PEGylated laccase" were applied, respectively. From this data, one can establish that the reaction improvement is more reliable if using PEGylated laccase because despite the similar DP, the enzyme is highly protected and gives a higher amount of polycatechol, as supported by powder quantification (data not shown). The PEG retained by the products of polymerization was detected in sample b) corresponding to the polymerization assisted by free PEG. If PEG remains even after several washing steps, it indicates that it is strongly complexed with the isolated products and there is no need of its elimination by an additional step.

\section{Conclusions}

Laccase from Myceliophthora thermophila was effective in the polymerization of catechol demonstrating to be an attractive alternative to conventional chemical processes. The role of poly(ethylene glycol) (PEG) as an additive in the enzymatic polymerization of catechol was evaluated. Higher polymerization degrees and an increased amount of polymer were achieved in the presence of PEG in the medium. The previous PEGylation of laccase showed a great potential for future applications because it allowed obtaining the same yields of polymerization as if free PEG is used, despite the lower specific activity resulted from PEGylation process. The novelty of this study relies on the effect of laccase PEGylation on the polymerization of polycatechol. A higher degree of polymerization as well as a higher amount polycatechol is obtained. The mobility was found to be a crucial property for the success of "template-as-

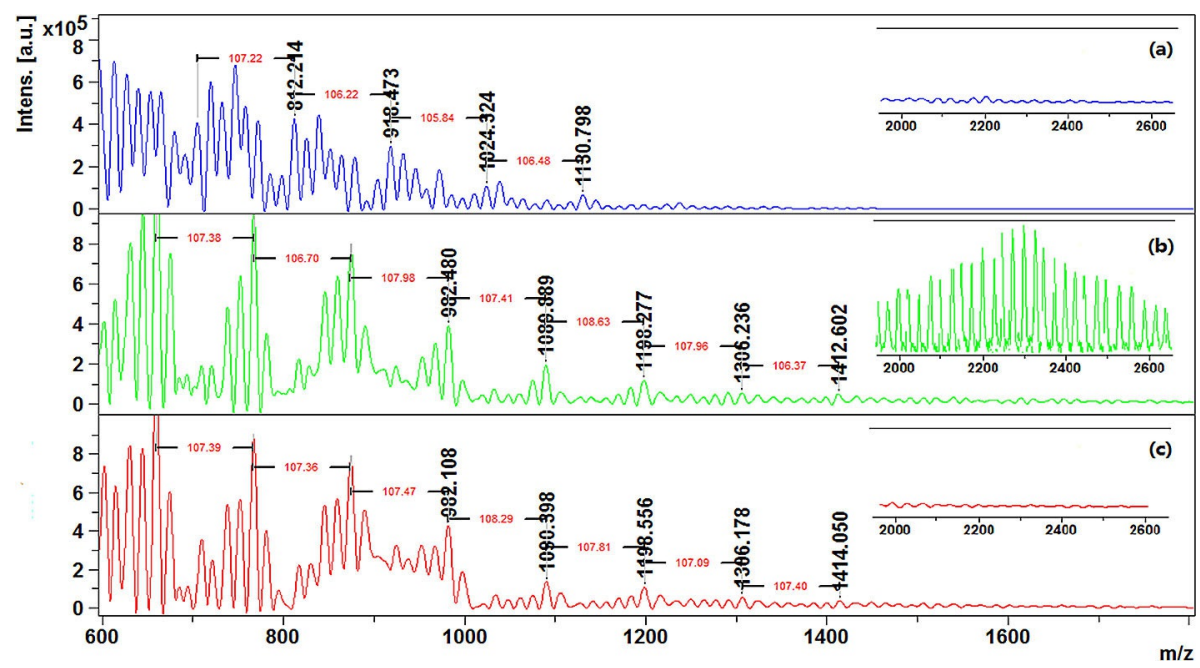

Figure 5. MALDI-TOF analysis of polycatechol polymerized by using (a) laccase; (b) laccase + PEG; (c) PEGylated laccase; the zoom area between 2000 and $3000 \mathrm{~m} / \mathrm{z}$ highlights the presence of PEG after polymerization. 
sisted" polymerization. The "PEG effect" is not perceptible after laccase entrapment because of the enzyme constraint if it is entrapped in polyacrylamide supports. The effect of PEG as a template is also highly dependent, as reported previously, ${ }^{[1]}$ on the type and structure of monomer. The polymeric structure of the additive and its interaction with the enzyme may be contributing factors to the template effect. As we have focused our study only on the polymerization of catechol, the effect of the PEG on the laccase-based polymerization of other compounds is hard to predict. Our data on laccase after ultrafiltration suggest that the PEG effect on crude enzymes might be reduced.

The mechanism of the PEG effect on laccase-based polymerization is not clearly understood, but molecular dynamics data suggest that PEG needs to interact (mix well) with the polymer formed. Here, a new environmentally friendly system was designed for the enzymatic polymerization of catechol opening space for further studies and optimization steps.

\section{Experimental Section}

Laccase from Myceliophthora thermophila was PEGylated by using the procedure reported by Daly et al. ${ }^{[22]}$ Briefly, $12 \mathrm{mg} \mathrm{mL}^{-1}$ laccase $(14.0 \mathrm{~mL})$ was reacted with $20 \mathrm{kDa}, \mathrm{O}$-[2-(6-oxocaproylamino)ethyl]$O^{\prime}$-methylpoly(ethylene glycol) (mPEG) at $\mathrm{pH} 5,10 \mathrm{~mm}$ sodium phosphate buffer with $20 \mathrm{~mm}$ sodium cyanoborohydride. A control reaction without $\mathrm{mPEG}$ was also conducted in each experiment. The reactions were stirred rapidly for $17 \mathrm{~h}$ at $4^{\circ} \mathrm{C}$. After $10 \mathrm{~min}$ mixing, the reagents were completely dissolved, and an aliquot was taken at time $0 \mathrm{~h}$ and after $17 \mathrm{~h}$ of incubation. These samples were ultrafiltrated by using a $30 \mathrm{kDa}$ cellulose membrane mounted in an ultrafiltration apparatus to separate the unbounded PEG. The PEGylated enzyme was then freeze-dried and analyzed by SDSPAGE. The samples for the SDS-PAGE analysis were assembled as follows: a $4 \mathrm{X}$ loading buffer $(0.001 \mathrm{~mL})$ was added to a $0.005 \mathrm{~mL}$ volume of the sample, mixed, and heated for $1 \mathrm{~min}$ at $98^{\circ} \mathrm{C}$ in a digital heat block. $10 \%$ acrylamide gels, containing $1 \%$ SDS were run at $40 \mathrm{~mA}$ for $60 \mathrm{~min}$ and silver-stained to visualize PEGylation.

\section{Enzymatic-assisted polymerization of catechol in solution}

Catechol polymerization was processed by incubating $10 \mathrm{mg} \mathrm{mL}^{-1}$ monomer in different solutions: a) $98 \mathrm{mg} \mathrm{mL}^{-1}$ native laccase; b) $98 \mathrm{mg} \mathrm{mL}^{-1}$ native laccase $+0.5 \mathrm{mg} \mathrm{mL}^{-1}$ PEG (3-4 kDa); and c) $98 \mathrm{mg} \mathrm{mL}^{-1}$ PEGylated laccase, in acetate buffer ( $\mathrm{pH}$ 5). The reactions were performed in a water bath at $40^{\circ} \mathrm{C}$ for $8 \mathrm{~h}$. Afterwards, the powder was washed with water by centrifugation and dried under vacuum for posterior MALDI-TOF analysis.

\section{Enzymatic-assisted polymerization of catechol in acrylamide gels}

A different approach for the polymerization of catechol was assessed by using laccase previously immobilized onto polyacrylamide gels. The polyacrylamide gels were produced by mixing acrylamide $(0.7 \mathrm{~mL}, 30 \%)$, Tris- $\mathrm{HCl}(1.3 \mathrm{~mL}, 0.5 \mathrm{M}, \mathrm{pH} 6.8)$, and distilled water $(2.97 \mathrm{~mL})$ in different well spots of a 6 -well microplate. Then, tetramethylethylenediamine (TEMED, $10 \mu \mathrm{L}$ ) and ammonium persulfate (APS, $10 \%, 37.5 \mu \mathrm{L}$ ) were added and mixed. Afterwards, the enzyme ( $98 \mathrm{mg} \mathrm{mL}^{-1}$ ) was added to each spot: native laccase; native laccase + PEG, and PEGylated laccase, and 30 min later, the gels were formed. A control gel without catalyst was also considered. After gels production, the polymerase activity of the entrapped catalyst was evaluated by adding $5 \mathrm{mg} \mathrm{mL}^{-1}$ catechol into the gels, and the reaction proceeded for $8 \mathrm{~h}$ at $40^{\circ} \mathrm{C}$.

\section{Determination of Total Content of Free $\mathrm{OH}$ groups}

The total content of free $\mathrm{OH}$ groups before and after polymerization was determined by using the Folin-Ciocalteu spectrophotometric method. The monomer and polymer solutions dissolved in DMSO $(100 \mu \mathrm{L})$ were added to the mixture of Folin-Ciocalteu reagent $(500 \mu \mathrm{L})$ and distilled water $(6 \mathrm{~mL})$, and the mixture was shaken for $1 \mathrm{~min}$. Then, $\mathrm{Na}_{2} \mathrm{CO}_{3}$ solution $(15 \%, 2 \mathrm{~mL})$ was added to the mixture and shaken for $1 \mathrm{~min}$. Later, the solution was brought up to $10 \mathrm{~mL}$ by adding distilled water. After $2 \mathrm{~h}$, the absorbance at $750 \mathrm{~nm}\left(25^{\circ} \mathrm{C}\right)$ was measured. The total content of free $\mathrm{OH}$ was assessed by plotting a gallic acid calibration curve (from 1 to $1500 \mu \mathrm{g} \mathrm{mL}^{-1}$ ). The equation of the gallic acid calibration curve was $A=0.2977 c+0.0368$, and the correlation coefficient was $r^{2}=0.9988$

\section{Polymers characterization}

The polymerization reactions were followed by UV/Vis spectroscopy using a 96-quartz microplate reader. The polymer products obtained were characterized by MALDI-TOF mass spectrometry. MALDI-TOF mass spectra were acquired on a Bruker Autoflex Speed instrument (Bruker Daltonics $\mathrm{GmbH}$ ) equipped with a $337 \mathrm{~nm}$ nitrogen laser. The matrix solution for MALDI-TOF measurements was prepared by dissolving a saturated solution of 2,5-dihydroxybenzoinc acid (DHB) in $100 \%$ ethanol. Samples were spotted onto a ground steel target plate (Bruker part $n^{\circ}$ 209519) and analyzed in the linear negative mode by using factory-configured instrument parameters suitable for a $1-10 \mathrm{kDa} \mathrm{m} / \mathrm{z}$ range (ion source 1: $19.5 \mathrm{kV}$; ion source 2: $18.3 \mathrm{kV}$ ). The time delay between laser pulse and ion extraction was set to $130 \mathrm{~ns}$, and the laser frequency was $25 \mathrm{~Hz}$.

\section{Calculation of average polymerization degree}

The $M_{\mathrm{n}}$ (number-average molecular weight) and $M_{\mathrm{w}}$ (weight-average molecular weight) of polycatechol obtained after oxidation was obtained by MALDI-TOF direct analysis and according to the equations

$$
\begin{aligned}
M_{n} & =\frac{\sum n_{i} M_{i}}{\sum n_{i}} \\
M_{w} & =\frac{\sum n_{i} M_{i}^{2}}{\sum n_{i} M_{i}} \\
P d & =\frac{M_{w}}{M_{n}}
\end{aligned}
$$

Where $n_{i}$ is the relative abundance of each peak; $M_{i}$ is the $\mathrm{m} / \mathrm{z}$ correspondent to each peak; $P d$ is the polydispersity.

\section{Molecular dynamics simulations}

All the simulations were performed with GROMACS 4.6, using GROMOS 54a7 force field. The molecules were parametrized by using the Automated Topology Builder (ATB). The system size was chosen according to the minimum image convention, taking into 
account a cutoff of $1.4 \mathrm{~nm}$. Nonbonded interactions were calculated by using a twin-range method, with short and long range cutoffs of 0.8 and $1.4 \mathrm{~nm}$, respectively. Neighbor searching was performed up to $1.4 \mathrm{~nm}$ and updated every fifth step. A time step of integration of $2 \mathrm{fs}$ was used. A reaction field correction for the electrostatic interactions was applied by using a dielectric constant of 54. The single-point-charge model was used for water molecules. The initial systems were energy minimized for 2000 steps by using the steepest descent method, with all heavy atoms harmonically restrained by using a force constant of $1000 \mathrm{~kJ} \mathrm{~mol}^{-1} \mathrm{~nm}^{-2}$. The systems were initialized in the canonical ensemble (NVT) for $50 \mathrm{~ns}$, with all heavy atoms harmonically restrained by using a force constant of $1000 \mathrm{~kJ} \mathrm{~mol}^{-1} \mathrm{~nm}^{-2}$. The simulation was then continued for $50 \mathrm{~ns}$ in the isothermal-isobaric ensemble (NPT), with the heavy atoms harmonically restrained with the same force constant. Pressure control was implemented using the Berendsen barostat, with a reference pressure of $1 \mathrm{bar}, 0.5 \mathrm{ps}$ of relaxation time, and isothermal compressibility of $4.5 \times 10^{5}$ bar. Temperature control was set using the V-rescale thermostat at $300 \mathrm{~K}$. The solutes and the solvent molecules were coupled in separated heat baths, with temperatures coupling constants of $0.025 \mathrm{ps}$ in the first two initialization steps and with $0.1 \mathrm{ps}$ for the rest of the simulations. The simulations were conducted for $10 \mathrm{~ns}$.

\section{Acknowledgements}

This study was supported by Chinese Government Scholarship under China Scholarship Council (No. 201606790036), Chinese Foundation Key projects of governmental cooperation in international scientific and technological innovation (No. 2016 YFE0115700), the National Natural Science Foundation of China (No.31470509) and the Fundamental Research Funds for the Central Universities (No.JUSRP51622A). This study was also supported by the Portuguese Foundation for Science and Technology (FCT) under the scope of the strategic funding of UID/BIO/04469/2013 unit and COMPETE 2020 (POCI-01-0145-FEDER-006684) and BioTecNorte operation (NORTE-01-0145-FEDER-000004) funded by European Regional Development Fund under the scope of Norte2020_-Programa Operacional Regional do Norte.

\section{Conflict of interest}

The authors declare no conflict of interest.
Keywords: alcohols - enzyme catalysis - molecular dynamics . polymerization $\cdot$ template synthesis

[1] A. Steevensz, M. M. Al-Ansari, K. E. Taylor, J. K. Bewtra, N. Biswas, J. Chem. Technol. Biotechnol. 2012, 87, $21-32$.

[2] Y.-J. Kim, H. Uyama, S. Kobayashi, Polym. J. 2004, 36, $992-998$.

[3] J. R. Jeon, E. J. Kim, K. Murugesan, H. K. Park, Y. M. Kim, J. H. Kwon, W. G. Kim, J. Y. Lee, Y. S. Chang, J. Microb. Biotechnol. 2010, 3, 324-335.

[4] S. Kim, C. Silva, D. V. Evtuguin, J. A. F. Gamelas, A. Cavaco-Paulo, Appl. Microbiol. Biotechnol. 2011, 89, $981-987$.

[5] R. M. Desentis-Mendoza, H. Hernandez-Sanchez, A. Moreno, R. D. C. Emilio, L. Chel-Guerrero, J. Tamariz, M. E. Jaramillo-Flores, Biomacromolecules 2006, 7, 1845-1854.

[6] M. Akashi, H. Ajiro in Encyclopedia of Polymeric Nanomaterials (Eds.: S. Kobayashi, K. Müllen), Springer, Berlin, Heidelberg, 2021, pp. 1-6.

[7] P. Walde, Z. Guo, Soft Matter 2011, 7, 316-331.

[8] Y.-J. Kim, H. Uyama, S. Kobayashi, Macromolecules 2003, 36, 5058-5060.

[9] R. Pilz, E. Hammer, F. Schauer, U. Kragl, Appl. Microbiol. Biotechnol. 2003, $60,708-712$

[10] S.-i. Shoda, H. Uyama, J.-i. Kadokawa, S. Kimura, S. Kobayashi, Chem. Rev. 2016, 116, 2307-2413.

[11] K. Modaressi, K. E. Taylor, J. K. Bewtra, N. Biswas, Water Res. 2005, 39, 4309-4316.

[12] J. Su, J. Fu, Q. Wang, C. Silva, A. Cavaco-Paulo, Crit. Rev. Biotechnol. 2017, 1-14.

[13] Y. J. Kim, J. A. Nicell, Bioresour. Technol. 2006, 97, 1431-1442.

[14] N. Durán, M. A. Rosa, A. D'Annibale, L. Gianfreda, Enzyme Microb. Technol. 2002, 31, 907-931.

[15] Y.-J. Kim, J. A. Nicell, Process Biochem. 2006, 41, 1029- 1037.

[16] A. Zerva, N. Manos, S. Vouyiouka, P. Christakopoulos, E. Topakas, Molecules 2016, 21, 550 .

[17] M. Fernández-Fernández, M. Á. Sanromán, D. Moldes, Biotechnol. Adv. 2013, 31, 1808-1825.

[18] A. Ursoiu, C. Paul, T. Kurtán, F. Péter, Molecules 2012, 17, 13045-13061.

[19] N. Aktaş, N. Şahiner, Ö. Kantoğlu, B. Salih, A. Tanyolaç, J. Polym. Environ. 2003, 11, 123-128.

[20] X. Qin, W. Xie, S. Tian, J. Cai, H. Yuan, Z. Yu, G. L. Butterfoss, A. C. Khuong, R. A. Gross, Chem. Commun. 2013, 49, 4839-4841.

[21] K. Mayolo-Deloisa, M. Gonzalez-Gonzalez, J. Simental-Martinez, M. RitoPalomares, J. Mol. Recognit. 2015, 28, 173-179.

[22] S. M. Daly, T. M. Przybycien, R. D. Tilton, Langmuir 2005, 21, 1328-1337.

Manuscript received: May 23, 2017

Revised manuscript received: June 19, 2017

Accepted manuscript online: June 22, 2017

Version of record online: September 29, 2017 